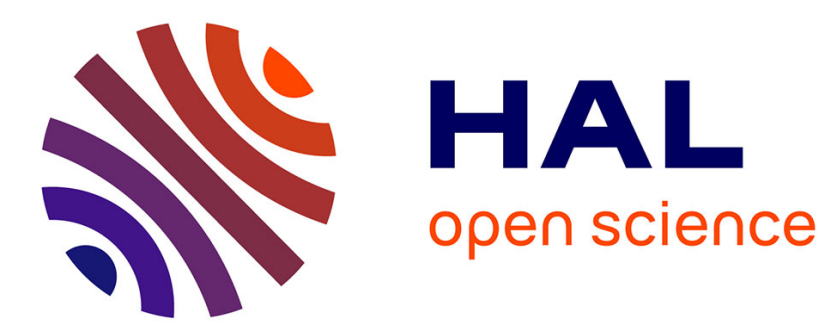

\title{
Modeling and Control of FAST-Hex: a Fully-Actuated by Synchronized-Tilting Hexarotor
}

\author{
Markus Ryll, Davide Bicego, Antonio Franchi
}

\section{To cite this version:}

Markus Ryll, Davide Bicego, Antonio Franchi. Modeling and Control of FAST-Hex: a Fully-Actuated by Synchronized-Tilting Hexarotor. IEEE/RSJ International Conference on Intelligent Robots and Systems ( IROS ) 2016, Oct 2016, Daejeon, South Korea. hal-01348538

\section{HAL Id: hal-01348538 \\ https://hal.science/hal-01348538}

Submitted on 28 Jul 2016

HAL is a multi-disciplinary open access archive for the deposit and dissemination of scientific research documents, whether they are published or not. The documents may come from teaching and research institutions in France or abroad, or from public or private research centers.
L'archive ouverte pluridisciplinaire HAL, est destinée au dépôt et à la diffusion de documents scientifiques de niveau recherche, publiés ou non, émanant des établissements d'enseignement et de recherche français ou étrangers, des laboratoires publics ou privés. 


\title{
Modeling and Control of FAST-Hex: a Fully-Actuated by Synchronized-Tilting Hexarotor
}

\author{
Markus Ryll ${ }^{1}$, Davide Bicego ${ }^{1}$ and Antonio Franchi ${ }^{1}$
}

\begin{abstract}
We present FAST-Hex, a novel UAV concept which is able to smoothly change its configuration from underactuated to fully actuated by using only one additional motor that tilts all propellers at the same time. FAST-Hex can adapt to the task at hand by finely tuning its configuration from the efficient (but underactuated) flight (typical of coplanar multirotor platforms) to the full-pose-tracking (but less efficient) flight, which is attainable by non-coplanar multi-rotors. We also introduce a novel full-pose geometric controller for generic multi-rotors (not only the FAST-Hex) that outperforms classical inverse dynamics approaches. The controller receives as input any reference pose in $\mathbb{R}^{3} \times \mathrm{SO}(3)$ (3D position + 3D orientation). Exact tracking is achieved if the reference pose is feasible with respect to the propeller spinning rate saturations. In case of unfeasibility a new feasible desired trajectory is generated online giving priority to the positional part. The new controller is tested with the FAST-Hex but can be used for many other multi-rotor platforms: underactuated, slightly fully-actuated and completely fully-actuated.
\end{abstract}

\section{INTRODUCTION}

Unmanned Aerial Vehicles (UAVs) are widely used in different application scenarios like remote monitoring and aerial photography, search and rescue missions and, more and more, aerial physical interaction with the environment [1], [2] and the with the human operator [3]. This new field of complex tasks including grasping and manipulation results in new challenges in the mechanical structure, the design and furthermore the control of aerial vehicles.

Recently, fully-actuated non-coplanar multi-rotor systems emerged as a valid solution to either benefit from a faster disturbance rejection [4], [5] or achieve a full-pose tracking, i.e., a decoupled tracking of a desired 3D position and 3D orientation [6]-[12]. Furthermore, fully actuated systems can be adapted to track a desired wrench and are therefore optimal tools in physical interaction tasks. The benefits come with the drawback of a reduced efficiency with respect to standard coplanar multi-rotors due to higher internal forces.

The first contribution of this paper is to present a new concept, the Fully-Actuated by Synchronized-Tilting Hexarotor (FAST-Hex), with six propellers actively tiltable by means of only one additional servomotor. With respect to solutions with more than one servomotor [5], [8], [10], [11] the use of a single one reduces the energy consumption, the total mass, the probability of failure, and the complexity of the system. Using this additional input, the platform can be driven along a continuum of configurations going from the energetically

\footnotetext{
${ }^{1}$ LAAS-CNRS, Université de Toulouse, CNRS, Toulouse, France, mryll@laas.fr, dbicego@laas.fr, afranchi@laas.fr

This work has been partially funded by the European Union's Horizon 2020 research and innovation programme under grant agreement No 644271 AEROARMS and the German Research Foundation (DFG).
}

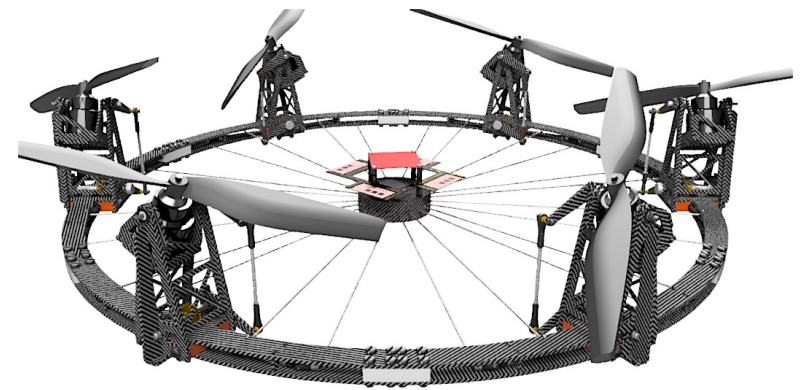

Fig. 1: Preliminary CAD prototype of the FAST-Hex concept.

efficient (but underactuated) one to the maximally actuated (but less efficient) one. The high-level fine tuning of the single tilting input allows to find the best trade-off between decoupled tracking and power efficiency for the task at hand. Therefore, from this point of view, the FAST-Hex clearly outperforms fixed non-coplanar multi-rotors [4], [6], [9], [12].

The second contribution is to introduce a new lowerlevel full-pose controller for the six propeller speeds that works seamlessly with any kind of multi-rotor platform (both coplanar and non-coplanar). The controller accepts as reference a full pose trajectory in $\mathbb{R}^{3} \times \mathrm{SO}(3)$ and modifies it (giving priority to the positional part) only when strictly needed by the limitation imposed by the spinning rate saturations of the six propellers. The controller finds its perfect application to the FAST-Hex as, differently from inverse dynamics approaches, it optimally deals with configurations that are slightly fully-actuated, like in the case of quasicoplanar multi-rotors. However, it is applicable to a much larger category of multi-rotors (beyond the FAST-Hex).

The paper is structured as follows. We describe and derive a dynamical model of the FAST-Hex in Sec. II. In Sec. III we develop a full-pose geometric control in $\mathbb{R}^{3} \times \mathrm{SO}(3)$ for generic multi-rotors and we test its validity in a realistic simulation in Sec. IV. Finally, Sec. V concludes the paper with a summary of the results and an outline of future work.

\section{Modeling}

The CAD model of a preliminary design of FAST-Hex is shown in Fig. 1. Its main feature is the ability to tilt the six propellers synchronously by using a transmission system connected to a single servomotor. In this section we introduce a simplified mathematical model that is used to derive the controller in Sec. III. A more accurate mathematical model will be used for the numerical validation of Sec. IV.

In its simplest model FAST-Hex is composed by a rigid body and six massless orientable propellers. We refer the 


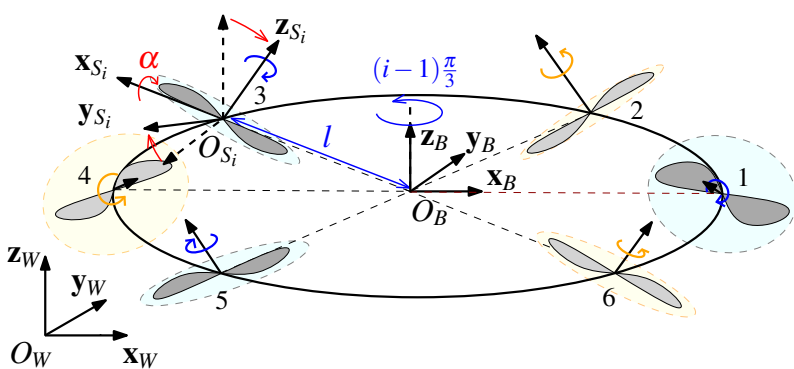

Fig. 2: A sketch of the simplified model of FAST-Hex. We highlighted all quantities related to the $(i=3)$-th propeller group. Clockwise spinning propellers $\{1,3,5\}$ are depicted in light-blue, while the counterclockwise spinning ones $\{2,4,6\}$ in light-orange.

reader to Fig. 2, where all main frames and symbols are reported. Let us define a world frame $\mathscr{F}_{W}=O_{W},\left\{\mathbf{x}_{W}, \mathbf{y}_{W}, \mathbf{z}_{W}\right\}$, the body frame $\mathscr{F}_{B}=O_{B},\left\{\mathbf{x}_{B}, \mathbf{y}_{B}, \mathbf{z}_{B}\right\}$, rigidly attached to the platform, where $O_{B}$ corresponds to the geometric center of the six propellers and to the center of mass (CoM) of the platform. The position of $O_{B}$ expressed in $\mathscr{F}_{W}$ is denoted by $\mathbf{p}_{B} \in \mathbb{R}^{3}$ and the orientation of $\mathscr{F}_{B}$ in $\mathscr{F}_{W}$ is represented by the rotation matrix $\mathbf{R}_{B} \in \mathrm{SO}(3)$. The angular velocity of $\mathscr{F}_{B}$ with respect to $\mathscr{F}_{W}$, expressed in $\mathscr{F}_{B}$, is denoted with $\boldsymbol{\omega}_{B} \in \mathbb{R}^{3}$. The kinematics of $\mathbf{R}_{B}$ is then

$$
\dot{\mathbf{R}}_{B}=\mathbf{R}_{B}\left[\boldsymbol{\omega}_{B}\right]_{\times},
$$

where $[\bullet]_{\times} \in \mathrm{so}(3)$ represents, in general, the skew symmetric matrix associated to any vector $\bullet \in \mathbb{R}^{3}$.

Hereafter we denote with $\mathbf{e}_{1}, \mathbf{e}_{2}$, and $\mathbf{e}_{3}$ the three vectors of the canonical basis of $\mathbb{R}^{3}$, and with $\mathbf{R}_{x}, \mathbf{R}_{y}$, and $\mathbf{R}_{z}$, the three canonical rotation matrices in $\mathrm{SO}(3)$.

Let us consider six frames $\mathscr{F}_{S_{1}}, \ldots, \mathscr{F}_{S_{6}}$, where $\mathscr{F}_{S_{i}}=$ $O_{S_{i}},\left\{\mathbf{x}_{S_{i}}, \mathbf{y}_{S_{i}}, \mathbf{z}_{S_{i}}\right\}$. The orientation of $\mathscr{F}_{S_{i}}$ with respect to $\mathscr{F}_{B}$ is represented by the rotation matrix

$$
\mathbf{R}_{S_{i}}^{B}(\alpha)=\mathbf{R}_{z}\left((i-1) \frac{\pi}{3}\right) \mathbf{R}_{x}\left((-1)^{i-1} \alpha\right), \quad i=1, \ldots, 6
$$

where $\alpha \in \mathscr{A}$ is the synchronized tilting angle which is adjustable by using a unique servomotor and a suitable transmission system (see Fig. 1 and attached video). The presence of $(-1)^{i-1}$ in (2) models the fact that the rotation axes of propellers with adjacent indexes are designed to tilt in an opposite fashion, which guarantees the full actuation of the platform for $\alpha \in \mathscr{A} \backslash\{0\}$, see, e.g., [9] for an explanation.

The vector from $O_{B}$ to $O_{S_{i}}$, describing the position of the center of the $i$-th propeller, expressed in $\mathscr{F}_{B}$, is

$$
\mathbf{p}_{B, S_{i}}^{B}=l \mathbf{R}_{z}\left((i-1) \frac{\pi}{3}\right) \mathbf{e}_{1} \quad \text { for } i=1, \ldots, 6
$$

where $l>0$ is the distance from $O_{B}$ to $O_{S_{i}}$.

The $i$-th propeller is centered at $O_{S_{i}}$ and rotates with angular velocity $(-1)^{i} w_{i} \mathbf{z}_{S_{i}}$ where $w_{i}>0$ is the controllable propeller spinning rate. While rotating, the propeller exerts simultaneously a thrust force and a drag moment, applied in $O_{S_{i}}$ and oriented along $\mathbf{z}_{S_{i}}$, that, expressed in $\mathscr{F}_{B}$, are

$$
\begin{aligned}
\mathbf{f}_{i}^{B}\left(f_{i}, \alpha\right) & =f_{i} \mathbf{R}_{S_{i}}^{B}(\alpha) \mathbf{e}_{3}, \quad \text { for } i=1, \ldots, 6, \quad \text { and } \\
\boldsymbol{\tau}_{i}^{B}\left(f_{i}, \alpha\right) & =(-1)^{i-1} c_{f}^{\tau} f_{i} \mathbf{R}_{S_{i}}^{B}(\alpha) \mathbf{e}_{3}, \quad \text { for } i=1, \ldots, 6,
\end{aligned}
$$

respectively, where $c_{f}^{\tau}>0$ is a constant parameter characteristic of the type of propeller, $f_{i}$ is the intensity of the force produced by the propeller, which is related to the controllable spinning rate by means of the quadratic relation

$$
f_{i}=c_{f} w_{i}^{2}
$$

where $c_{f}>0$ is another propeller-dependent constant parameter. The presence of $(-1)^{i}$ in (2) and (5) models the fact that propellers with adjacent indexes are designed to spin with opposite sign and thus generate opposite drags.

Summing all the thrust forces we can compute the total force applied to the platform in its CoM, expressed in $\mathscr{F}_{W}$

$$
\mathbf{f}^{W}(\alpha, \mathbf{u})=\mathbf{R}_{B} \sum_{i=1}^{6} \mathbf{f}_{i}^{B}\left(f_{i}, \alpha\right)=\mathbf{R}_{B} \mathbf{F}_{1}(\alpha) \mathbf{u},
$$

where $\mathbf{u}=\left[f_{1} f_{2} f_{3} f_{4} f_{5} f_{6}\right]^{T}$ and $\mathbf{F}_{1}(\alpha) \in \mathbb{R}^{3 \times 6}$ is a suitable $\alpha$-dependent matrix. If $\alpha=0$, i.e., all the propellers are coplanar like in a standard hexarotor, then $\mathbf{F}_{1}(\alpha)=\left[\begin{array}{lll}\mathbf{0}_{6} & \mathbf{0}_{6} & \mathbf{1}_{6}\end{array}\right]^{T}$.

Summing all moment contributions (drag moments and thrust contributions), we obtain the total moment applied to the platform, with respect to $O_{B}$, and expressed in $\mathscr{F}_{B}$

$$
\tau^{B}(\alpha, \mathbf{u})=\sum_{i=1}^{6}\left(\left(\mathbf{p}_{B, S_{i}}^{B} \times \mathbf{f}_{i}^{B}\left(f_{i}, \alpha\right)\right)+\boldsymbol{\tau}_{i}^{B}\left(f_{i}, \alpha\right)\right)=\mathbf{F}_{2}(\alpha) \mathbf{u}
$$

Using the Newton-Euler approach, the equations of motion of the aerial platform can be then compactly written as

$$
\left[\begin{array}{c}
m \ddot{\mathbf{p}}_{B} \\
\mathbf{J} \dot{\boldsymbol{\omega}}_{B}
\end{array}\right]=-\left[\begin{array}{c}
m g \mathbf{e}_{3} \\
\boldsymbol{\omega}_{B} \times \mathbf{J} \boldsymbol{\omega}_{B}
\end{array}\right]+\left[\begin{array}{c}
\mathbf{f}^{W} \\
\boldsymbol{\tau}^{B}
\end{array}\right]
$$

where $\mathbf{J}>0$ is the $3 \times 3$ inertia matrix of the rigid body with respect to $O_{B}$ and expressed in $\mathscr{F}_{B}, m>0$ is the total mass of the platform, and $g>0$ is the gravitational acceleration.

Replacing (7) and (8) in (9) we obtain

$$
\left[\begin{array}{c}
m \ddot{\mathbf{p}}_{B} \\
\mathbf{J} \dot{\boldsymbol{\omega}}_{B}
\end{array}\right]=-\left[\begin{array}{c}
m g \mathbf{e}_{3} \\
\boldsymbol{\omega}_{B} \times \mathbf{J} \boldsymbol{\omega}_{B}
\end{array}\right]+\underbrace{\left[\begin{array}{c}
\mathbf{R}_{B} \mathbf{F}_{1}(\alpha) \\
\mathbf{F}_{2}(\alpha)
\end{array}\right]}_{\mathbf{F}\left(\mathbf{R}_{B}, \alpha\right)} \mathbf{u} .
$$

Finally, we take into account the propeller spinning rate saturations, that can be expressed as input saturations as

$$
\mathbf{u} \in \mathscr{U}=\left\{\mathbf{u} \in \mathbb{R}^{6} \mid 0 \leq \underline{u} \leq f_{i} \leq \bar{u} \quad \forall i=1 \ldots 6\right\} .
$$

A. Synchronized Tilting Angle: Efficiency vs. Full-Actuation

FAST-Hex has two structurally different configurations:

1) $\alpha=0 \Rightarrow \operatorname{rank}\left(\mathbf{F}\left(\mathbf{R}_{B}, \alpha=0\right)\right)=4$

2) $\alpha \in \mathscr{A} \backslash\{0\} \Rightarrow \operatorname{rank}\left(\mathbf{F}\left(\mathbf{R}_{B}, \alpha\right)\right)=6$.

In configuration 1) all propellers of the FAST-Hex are coplanar or, equivalently, their spinning axes are all collinear. The system degenerates to an ordinary hexarotor platform. In this configuration the internal forces during hovering are zero. Only internal torques due to the drag moment appear. These are typically one order of magnitude less strong than the torques generated by the thrust moments and therefore neglected in the following efficiency considerations.

We model the wasted force using the following index

$$
\eta_{f}(\alpha, \mathbf{u})=\frac{\left\|\sum_{i=1}^{6} \mathbf{f}_{i}^{B}\left(f_{i}, \alpha\right)\right\|}{\sum_{i=1}^{6}\left\|\mathbf{f}_{i}^{B}\left(f_{i}, \alpha\right)\right\|}=\frac{\left\|\sum_{i=1}^{6} \mathbf{f}_{i}^{B}\left(f_{i}, \alpha\right)\right\|}{\sum_{i=1}^{6} f_{i}} \in[0,1]
$$




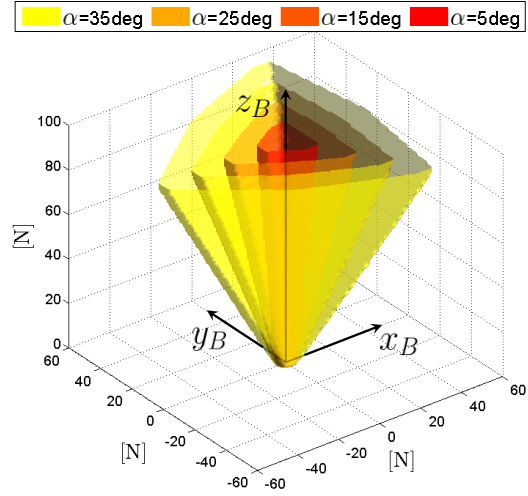

Fig. 3: Volume of attainable total forces $\mathbf{R}_{B}^{T} \mathbf{f}^{W}(\alpha, \mathbf{u})$ corresponding to different values of $\alpha$. The surfaces are computed using (7), expressed in the body frame $\mathscr{F}_{B}$, and imposing $f_{i}>0 \forall i=1, \ldots, 6$. The larger $\alpha$ the larger the volume of the pseudo-cone. For $\alpha=0$ the cone degenerates to a single direction along the $\mathbf{z}_{B}$ axis (not shown in the plot).

that we call the force efficiency index.

It is easy to check that $\eta_{f}(\alpha=0, \mathbf{u})=1$ for any input $\mathbf{u}$, which corresponds to maximum efficiency. Hence the configuration 1) is energetically very efficient. This comes with the drawback that the platform is underactuated and a simultaneous tracking of fully independent $\mathbf{p}_{r}(t)$ and $\mathbf{R}_{r}(t)$ is impossible. The best choice left in this case is a control that selects a new reference orientation, denoted with $\mathbf{R}_{d}(t)$, that is compatible ${ }^{1}$ with $\mathbf{p}_{r}(t)$ and is as close a possible to $\mathbf{R}_{r}(t)$ with respect to a certain criterion, as, e.g., possessing the same yaw angle of $\mathbf{R}_{r}(t)$, or the same projection of a certain axis on a certain plane. This approach is used, e.g., by the well established geometric control [13], whose rotational part is based on [14]. Global convergence is achieved without the singularities of other orientation parametrization.

In the configurations of type 2) the internal forces in hovering are greater than zero, which means that the system is wasting more energy than in configuration 1). The larger $|\alpha|$ the larger the internal forces. This is clearly visible from the fact that $\eta_{f}(\alpha \in \mathscr{A} \backslash\{0\}, \mathbf{u})<1$. In particular, during horizontal hovering, when all the propellers are spinning at the same speed producing the same force $f$, we have that $\eta_{f}\left(\alpha, f \mathbf{1}_{6 \times 1}\right)=\cos \alpha$. If the platform is following a nonhovering trajectory then $\eta_{f}(\alpha, \mathbf{u})$ is in general different from $\cos \alpha$ and one has to use (12) to exactly compute it. On the other side in configurations of type 2) the platform is fully actuated, and the larger $|\alpha|$ the larger the volume of admissible total forces $\mathbf{f}^{W}$ in (9), as it can be clearly seen from Fig. 3. The simultaneous tracking of $\mathbf{p}_{r}(t)$ and $\mathbf{R}_{r}(t)$ becomes feasible as shown in [9], where a controller for this particular case is also proposed.

Due to the fact that $\alpha$ is a slowly changeable parameter, the change of $\alpha$ is delegated to a high-level slow-rate controller/planner or to a human operator. The high-level controller can gently tune $\alpha$ while flying, thus continuously changing the platform between configuration 1) and any of

\footnotetext{
${ }^{1}$ Compatibility is related to the well-known differential flatness property of coplanar-rotor vehicles. In particular, the $\mathbf{z}_{B}$ axis must be kept parallel to $\ddot{\mathbf{p}}_{r}(t)+m g \mathbf{e}_{3}$. The orientation about $\mathbf{z}_{B}$ is instead not constrained by $\mathbf{p}_{r}(t)$.
}

the configurations of type 2) in order to adapt to the particular task being executed. For example configuration 1) can be chosen when a pure horizontal hovering is requested while a type 2) configuration can be selected when hovering with non-zero roll and pitch is needed.

\section{Full-Pose GeOMEtric Control With PRIORITIZED POSITION TRACKING}

In this section we consider the problem of designing a low-level control law for the six spinning rate inputs $\mathbf{u}$ that lets $\mathbf{p}_{B}^{W}$ and $\mathbf{R}_{B}$ track at best an arbitrary full-pose reference trajectory $\left(\mathbf{p}_{r}(t), \mathbf{R}_{r}(t)\right): \mathbb{R} \rightarrow \mathbb{R}^{3} \times \mathrm{SO}(3)$. For the reasons given above, the low-level controller assumes that $\alpha$ is given. By decoupling the control of $\alpha$ and $\mathbf{u}$ we also make the lowlevel controller described in this section applicable to many other platforms beyond its specific use for the FAST-Hex.

One option to design the controller would be to use the geometric control [13] in configuration 1) and the fully actuated controller [9] in configuration 2). A part from the problems possibly generated by the use of a switching controller, this solution is not advisable as the computation of $\mathbf{F}\left(\mathbf{R}_{B}, \alpha\right)^{-1}$ (used in [9]) is ill-conditioned if $\alpha \rightarrow 0$ and it would result in very high control inputs which do not fulfill (11). The use of such controller on FAST-Hex would lead to saturations, unpredictable behavior and possible destabilization.

A possible remedy could be to use the geometric control [13] even for small values of $|\alpha|$, which would mean to give up on the possibility of tracking the full-pose trajectory when $\alpha$ is too small, i.e., a bad exploitation of the FASTHex full-actuation capabilities. In fact, driving the platform with $\alpha$ close to 0 is actually desirable, since in this case we might have the perfect balance between full actuation and minimization of the wasted internal forces.

Driven by these considerations, we propose to use a unique controller that works seamlessly in configurations of both types ((type 1) and (type 2)). The main design idea is to extend the underactuated geometric control [13] to the fullyactuated case. The resulting behavior of FAST-Hex with our novel controller will then be the following:

- the smaller $|\alpha|$ (and the larger $\left.\left\|\ddot{\mathbf{p}}_{r}(t)\right\|\right)$ the more the output of the control law resembles [13]. In other words, when $|\alpha|$ decreases FAST-Hex becomes gradually underactuated, i.e., it still keeps a good tracking of the reference position but it becomes progressively unable to independently track also the reference orientation;

- the larger $|\alpha|$ (and the smaller $\left\|\ddot{\mathbf{p}}_{r}(t)\right\|$ ) the more the controller generalizes [13], and FAST-Hex becomes gradually fully-actuated, i.e., more and more able to control simultaneously the position and the orientation in an independent way.

This approach is formally described in the following.

Similarly to [13], and to many other aerial vehicle controllers, our controller is composed by an inner control loop (the attitude controller) and outer control loop (the position controller), see Fig. 4 for a block diagram of the full control system. The controller terminates with a wrench mapper that computes the actual input $\mathbf{u}$ based on the reference control force $\mathbf{f}_{r} \in \mathbb{R}^{3}$ and reference control moment $\boldsymbol{\tau}_{r} \in \mathbb{R}^{3}$ provided 


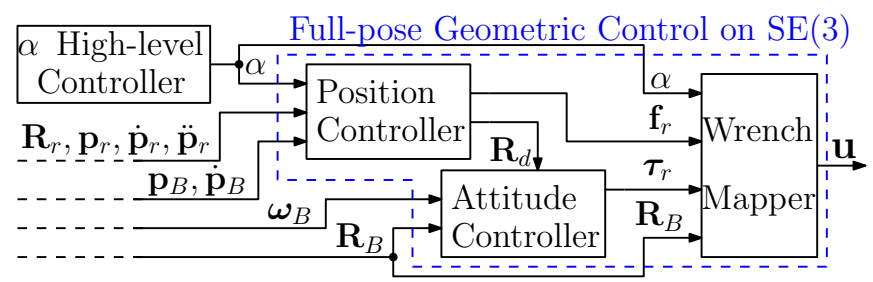

Fig. 4: Block diagram of the controller.

by the position and attitude controller, respectively. We shall describe in detail the three components in the following.

1) Attitude controller: The attitude controller is standard: it takes as input a desired orientation, denoted with $\mathbf{R}_{d} \in$ $S O(3)$, and the measured attitude state $\mathbf{R}_{B}, \boldsymbol{\omega}_{B}$ to compute the reference control torque $\boldsymbol{\tau}_{r} \in \mathbb{R}^{3}$ as

$$
\tau_{r}=\boldsymbol{\omega}_{B} \times \mathbf{J} \boldsymbol{\omega}_{B}-\mathbf{K}_{R} \mathbf{e}_{R}-\mathbf{K}_{\omega} \boldsymbol{\omega}_{B}
$$

where $\mathbf{K}_{R}, \mathbf{K}_{\omega}$ are positive gain matrixes, $\mathbf{e}_{R}$ is the orientation error defined as

$$
\mathbf{e}_{R}=\frac{1}{2}\left(\mathbf{R}_{d}^{T} \mathbf{R}_{B}-\mathbf{R}_{B}^{T} \mathbf{R}_{d}\right)^{\vee}
$$

and $\bullet \vee$ is the vee map from $s o(3)$ to $\mathbb{R}^{3}$, see, e.g., [13]. The vector $\tau_{r}$ is then passed to the wrench mapper. Notice that the desired orientation $\mathbf{R}_{d}$ is not in general the same as the reference orientation $\mathbf{R}_{r}$.

2) Position controller: The position controller takes as input the full-pose reference trajectory $\left(\mathbf{p}_{r}(t), \mathbf{R}_{r}(t)\right)$ and the measured translational state $\mathbf{p}_{B}, \dot{\mathbf{p}}_{B}$ and produces as output the desired orientation $\mathbf{R}_{d}$ (given as input to the attitude controller) and the reference control force $\mathbf{f}_{r} \in \mathbb{R}^{3}$ (sent directly to the wrench mapper).

In order to describe the position controller algorithm we start by defining the position tracking errors

$$
\mathbf{e}_{p}=\mathbf{p}_{B}-\mathbf{p}_{r}, \quad \text { and } \quad \mathbf{e}_{v}=\dot{\mathbf{e}}_{p}=\dot{\mathbf{p}}_{B}-\dot{\mathbf{p}}_{r} .
$$

from which the reference force vector is computed as

$$
\mathbf{f}_{r}=m\left(\ddot{\mathbf{p}}_{r}+g \mathbf{e}_{3}-\mathbf{K}_{p} \mathbf{e}_{p}-\mathbf{K}_{v} \mathbf{e}_{v}\right),
$$

where $\mathbf{K}_{p}$ and $\mathbf{K}_{v}$ are positive diagonal gain matrixes. It is easy to check that if $\mathbf{f}_{r}$ could be applied to the CoM of FASTHex then $\mathbf{e}_{p}$ would converge exponentially to zero with a dynamics dictated by $\mathbf{K}_{p}$ and $\mathbf{K}_{v}$.

Given $\alpha$, the set of orientations ${ }^{2}$ of the main body that allow to exert $\mathbf{f}_{r}$ on the CoM while satisfying the input constraints (11) can be defined recalling (7) and (8)

$$
\begin{aligned}
\mathscr{R}_{\alpha}\left(\mathbf{f}_{r}\right) & =\{\mathbf{R} \in S O(3) \mid \\
& \left.\exists \mathbf{u} \in \mathscr{U}, \mathbf{R F}_{1}(\alpha) \mathbf{u}=\mathbf{f}_{r} \wedge \mathbf{F}_{2}(\alpha) \mathbf{u}=\mathbf{0}\right\} .
\end{aligned}
$$

Simultaneous tracking of both $\mathbf{p}_{r}(t)$ and $\mathbf{R}_{r}(t)$ is possible iff $\mathbf{R}_{r}(t) \in \mathscr{R}_{\alpha}\left(\mathbf{f}_{r}(t)\right)$. In that case the position controller just selects $\mathbf{R}_{d}=\mathbf{R}_{r}$. Otherwise, our strategy is to modify $\mathbf{R}_{r}$ as less as possible in order to find an $\mathbf{R}_{d}$ that is contained in $\mathscr{R}_{\alpha}\left(\mathbf{f}_{r}(t)\right)$ and is also close to $\mathbf{R}_{r}$.

\footnotetext{
${ }^{2}$ This set can also be defined for a generic multi-rotor platform, thus allowing to extend our method beyond its specific use for the FAST-Hex.
}

In order to describe how $\mathbf{R}_{d}$ is computed in the FAST-Hex case let us define the following set of orientations

$$
\overline{\mathscr{R}}\left(\mathbf{f}_{r}\right)=\left\{\mathbf{R} \in S O(3) \mid \mathbf{R e}_{3} \times \mathbf{f}_{r}=0\right\},
$$

which represents the set of body orientations for which $\mathbf{z}_{B}$ is parallel to $\mathbf{f}_{r}$. It is easy to be convinced that, thanks to the way FAST-Hex is designed, the following relation holds:

$$
\mathscr{R}_{\alpha}\left(\mathbf{f}_{r}\right) \neq \emptyset \Rightarrow \mathscr{R}_{\alpha}\left(\mathbf{f}_{r}\right) \supset \overline{\mathscr{R}}\left(\mathbf{f}_{r}\right) .
$$

In fact, the direction $\mathbf{z}_{B}$ is the one along which the largest force can be exerted by the FAST-Hex.

Notice that if $\alpha=0$ then $\mathbf{z}_{B}$ is actually the only direction along which a force can be exerted, i.e.:

$$
\mathscr{R}_{\alpha=0}\left(\mathbf{f}_{r}\right) \neq \emptyset \Rightarrow \mathscr{R}_{\alpha=0}\left(\mathbf{f}_{r}\right)=\overline{\mathscr{R}}\left(\mathbf{f}_{r}\right) \text {. }
$$

When $\alpha>0$, the set $\mathscr{R}_{\alpha}\left(\mathbf{f}_{r}\right)$ may contain orientations in the 'neighborhood' of $\overline{\mathscr{R}}\left(\mathbf{f}_{r}\right)$. The main idea is then to search for $\mathbf{R}_{d}$ by moving from $\mathbf{R}_{r}$ toward the set $\overline{\mathscr{R}}\left(\mathbf{f}_{r}\right)$ and to stop as soon as an orientation that belongs to $\mathscr{R}_{\alpha}\left(\mathbf{f}_{r}\right)$ is found. In the best case $\left(\mathbf{R}_{r}(t) \in \mathscr{R}_{\alpha}\left(\mathbf{f}_{r}(t)\right)\right)$ the search stops immediately and the controller selects $\mathbf{R}_{d}=\mathbf{R}_{r}$; in the worst case the search terminates in $\overline{\mathscr{R}}\left(\mathbf{f}_{r}\right)$; in the general case it terminates somewhere in the middle.

In order to efficiently implement the search consider the rotation matrix $\mathbf{R}_{s}(\lambda)$ defined as the matrix that represents a rotation of an angle $\lambda$ about the vector $\mathbf{R}_{r} \mathbf{e}_{3} \times \mathbf{f}_{r}$. Then define $\lambda_{d}$ as the solution of

$$
\min _{\substack{\mathbf{R}_{s}(\lambda) \mathbf{R}_{r} \in \mathscr{R}_{\alpha}\left(\mathbf{f}_{r}\right) \\ \lambda \in\left[0, \lambda_{\max }\right]}} \lambda,
$$

where $\lambda_{\max }=\arccos \left(\frac{\mathbf{f}}{r}_{\left\|\mathbf{f}_{r}\right\|}^{T} \mathbf{R}_{r} \mathbf{e}_{3}\right)$ (i.e., $\lambda_{\max }$ is such that $\left.\mathbf{R}_{s}\left(\lambda_{\max }\right) \mathbf{R}_{r} \in \overline{\mathscr{R}}\left(\mathbf{f}_{r}\right)\right)$ and finally compute the desired orientation as

$$
\mathbf{R}_{d}=\mathbf{R}_{s}\left(\lambda_{d}\right) \mathbf{R}_{r} .
$$

The minimization problem (21) is efficiently implemented using a bisection search on $\lambda \in\left[0, \lambda_{\max }\right]$.

3) Wrench mapper: The wrench mapper takes as inputs $\mathbf{f}_{r}$ and $\boldsymbol{\tau}_{r}$ and computes a feasible $\mathbf{u}$ using:

$$
\mathbf{u}=\left[\begin{array}{c}
\mathbf{R}_{B} \mathbf{F}_{1}(\alpha) \\
\mathbf{F}_{2}(\alpha)
\end{array}\right]^{-1}\left[\begin{array}{c}
\mathbf{f}_{c} \\
\boldsymbol{\tau}_{r}
\end{array}\right]
$$

Notice that (23) does not include directly $\mathbf{f}_{r}$, but it uses instead the vector $\mathbf{f}_{c} \in \mathbb{R}^{3}$. The vector $\mathbf{f}_{c}$ is computed by initializing it as $\mathbf{f}_{r}$ and then rotating it about $\mathbf{f}_{r} \times \mathbf{R}_{B} \mathbf{e}_{3}$ of the minimum angle that is needed to let $\mathbf{u}$ satisfy the constraints (11) or to eventually be parallel to $\mathbf{R}_{B} \mathbf{e}_{3}$. This computation is also efficiently implemented using a bisection search, as it is done for $\mathbf{R}_{d}$.

To sum up the full controller in words, we aim at tracking a desired full-pose reference trajectory $\left(\mathbf{p}_{r}(t), \mathbf{R}_{r}(t)\right)$ : if this is not feasible as the control output $\mathbf{u}$ would violate the minimum or maximum forces of any propeller $u_{i}$, we seek to find an $\mathbf{R}_{d}(t) \in \mathbf{R}_{\alpha}\left(\mathbf{f}_{r}\right)$ such that (11) is obeyed. Finally, since $\mathbf{R}_{d}$ cannot, in general, be attained instantaneously, we search for an $\mathbf{f}_{c}$ close to $\mathbf{f}_{r}$ that obeys (11) given the current orientation and we compute $\mathbf{u}$ using (23). 
TABLE I: Parameters of the accurate model used in the simulations.

\begin{tabular}{lll}
\hline Definition & Symbol & Value in Sim. \\
\hline Total mass of the structure without the propeller groups & $m_{S}$ & $1.5 \mathrm{Kg}$ \\
Mass of the $t^{t h}$ propeller group & $m_{i}$ & $0.15 \mathrm{Kg}$ \\
Mass of the whole FAST-Hex & $m$ & $2.4 \mathrm{Kg}$ \\
Gravity acceleration & $g$ & $9.81 \mathrm{~m} / \mathrm{s}^{2}$ \\
Distance between $O_{B}$ and $O_{S_{i}}$ & $l$ & $0.315 \mathrm{~m}$ \\
Parameter of the propeller linking $w_{i}$ to $f_{i}$ & $c_{f}$ & $6.3 * 10^{-4} \frac{\mathrm{N}}{\mathrm{Hz}^{2}}$ \\
Constant parameter characteristic of the propeller & $c_{f}^{\tau}$ & $1.7 * 10^{-2} \mathrm{~m}$ \\
\hline
\end{tabular}

\section{Simulations with an Accurate Model}

In this section we present simulation results to validate the presented controller. The model of the FAST-Hex introduced in Sec. II is useful in order to introduce the mathematical framework and to design the controller, but it is not realistic enough to predict the real behavior of the controlled platform in simulation. Therefore we developed a more advanced model which includes all the characteristics of the preliminary prototype depicted in Fig. 1 including but not limited to: physical properties obtained from the CAD model, control frequencies, delays, dynamics of the rotor spinning velocity control, tilting propeller groups with moving masses, change of the inertia matrix and the center of mass. A list of the main parameters used in the simulations can be found in Table I. Additionally, the following parameters have been also used: inertia tensor of the main body $\left.\mathbf{J}_{B}^{B}=\left[\begin{array}{lll}4.30 & 0 & 0\end{array}\right]^{T}\left[\begin{array}{lll}0 & 4.11 & 0\end{array}\right]^{T}\left[\begin{array}{lll}0 & 0 & 8.20\end{array}\right]^{T}\right]$. $10^{-2} \mathrm{Kg} / \mathrm{m}^{2}$; inertia tensor of the propeller group $\mathbf{J}_{P_{i}}^{P_{i}}=$ $\left.\left[\begin{array}{llll}1.97 & 0 & 0\end{array}\right]^{T}\left[\begin{array}{llll}0 & 1.97 & 0\end{array}\right]^{T}\left[\begin{array}{lll}0 & 0 & 1.21\end{array}\right]^{T}\right] \cdot 10^{-4} \mathrm{Kg} / \mathrm{m}^{2}$. Finally, the controller gains in (13) and (16) are chosen as $\mathbf{K}_{r}=70 \mathbf{I}_{3}$, $\mathbf{K}_{\omega}=10 \mathbf{I}_{3}, \mathbf{K}_{p}=45 \mathbf{I}_{3}, \mathbf{K}_{v}=15 \mathbf{I}_{3}$.

The results of a representative simulation are shown in Fig. 5 while additional simulation results can be seen in the attached video. For the reader's convenience the orientations corresponding to a rotation matrix used in the controller are displayed in the plots using the roll, pitch, and yaw angles with the following naming convention: $\mathbf{R}_{\bullet} \rightarrow \phi_{\bullet}, \theta_{\bullet}, \psi_{\bullet}$. The full-pose reference trajectory is defined by the position $\mathbf{p}_{r}(t)=\left[p_{r_{x}}(t) p_{r_{y}}(t) p_{r_{z}}(t)\right]^{T}$ and the orientation $\mathbf{R}_{r}(t)$, with $t \in[0,40] \mathrm{s}$. The trajectory $\mathbf{p}_{r}(t), \mathbf{R}_{r}(t)$ is chosen purposely unfeasible for a standard (co-planar) hexarotor (i.e., the FAST-Hex with $\alpha=0$ ). In particular, the position trajectory is constant w.r.t time for $p_{r_{y}}(t)=0 \mathrm{~m}$ and $p_{r_{z}}(t)=0.75 \mathrm{~m}$ and sinusoidal for $p_{r_{x}}(t)$. In a similar fashion the rotational trajectory is constant for roll and yaw $\left(\phi_{r}(t)=0 \mathrm{deg}\right.$ and $\left.\psi_{r}(t)=0 \mathrm{deg}\right)$ and sinusoidal for the pitch $\theta_{r}(t)$. The main parameters of the two sinusoids are given in Table II. Furthermore, the two sinusoids are out of phase by $180 \mathrm{deg}$ (see Fig. 5 - compare $p_{r_{x}}$ and $\theta_{r}$ ). In this way the FAST-Hex is tasked to change the direction of motion in position while flying backwards - a clearly unfeasible motion for co-planar multi-rotors. For further visualization we refer the reader to the attached video.

Concerning the signal $\alpha(t)$, we assume that $\alpha=0 \mathrm{deg}$ (underactuated configuration) for $t<15.2 \mathrm{~s}$; $\alpha$ is linearly increasing from $0 \mathrm{deg}$ to $35 \mathrm{deg}$ for $15.2 \mathrm{~s} \leq t \leq 30.2 \mathrm{~s}$; and that $\alpha=35 \mathrm{deg}$ for $t>30.2 \mathrm{~s}$ (largely fully-actuated configuration) (see Fig. 5- $\alpha$ ).
TABLE II: Features of $\mathbf{p}_{r}(t), \mathbf{R}_{r}(t)$ concerning $p_{r_{x}}(t)$ and $\theta_{r}(t)$

\begin{tabular}{lll}
\hline & Position $\left(p_{r_{x}}(t)\right)$ & Orientation $\left(\theta_{r}(t)\right)$ \\
\hline Amplitude: & $1 \mathrm{~m}$ & $10 \mathrm{deg}$ \\
Peak velocity: & $1 \mathrm{~m} / \mathrm{s}$ & $10 \mathrm{deg} / \mathrm{s}$ \\
Peak acceleration: & $1 \mathrm{~m} / \mathrm{s}^{2}$ & $10 \mathrm{deg} / \mathrm{s}^{2}$ \\
\hline
\end{tabular}

\section{A. Main findings}

1) Phases induced by a changing $\alpha$ : It becomes clear from the results that the trajectory tracking can be sub grouped into three phases, named underactuated phase, transition phase and fully actuated phase (see Fig. 5). The system is underactuated as long as $\alpha=0$. In this phase only the reference position can satisfyingly be tracked (maximum position error $\left\|\mathbf{e}_{p}\right\| \approx 0.05 \mathrm{~m}$ ) while the orientation is actually in opposition of phase with respect to the reference trajectory because the controller gives priority the position tracking. The second phase is determined by small values of $\alpha>0$ which allows a partial tracking of the reference orientation trajectory. In fact, although $\alpha>0$, the admissible force space (compare Fig. 3) is not always wide enough such that $\mathbf{f}_{r}$ in (16) remains inside. In these cases $\mathbf{R}_{r} \neq \mathbf{R}_{d}$ and $0<\lambda<\lambda_{\max }$. The last phase, the fully actuated phase, is characterized by a $\alpha>0$ that is large enough to let $\mathbf{f}_{r}$ always remain in the feasible set, resulting in a good simultaneous tracking of $\mathbf{p}_{r}$ and $\mathbf{R}_{r}$.

2) Trajectory tracking: It is very interesting to point out that the position tracking (see Fig. $5-e_{p_{x}}$ ) starts to improve in the transition phase and is even more improved in fully actuated phase compared to the underactuated phase. This effect is two-folded. It is clear that in the case of the underactuated system the position tracking is limited by the accuracy of the orientation tracking, while the tracking of both position and orientation start to become independent when $\alpha>0$. Additionally we would like to point out that the reference trajectory is currently only defined in $\mathbf{R}_{r}$ but its derivatives are not used (i.e., $\boldsymbol{\omega}_{r}, \dot{\boldsymbol{\omega}}_{r}$ ). How to correctly use $\left(\boldsymbol{\omega}_{r}, \dot{\boldsymbol{\omega}}_{r}\right)$ in the controller is not an easy problem to solve and an interesting subject of future research.

3) Force efficiency: The force efficiency index $\eta_{f}$ (presented in (12)) in Fig 5 shows the expected decreasing of the efficiency for $\alpha>0$. We would like to note that even for a high $\alpha=35 \mathrm{deg}$ the efficiency is only reduced by $\approx 18 \%$, which corresponds to an average of about $1 \mathrm{~N}$ more of thrust for each propeller w.r.t. the the underactuated case. Furthermore it is important to note that the actuation of the propeller groups (additional mechanics + motor) increases the mass of the full platform by $\approx 10 \%$. This additional efficiency loss has to be taken into account in the design phase in addition to $\eta_{f}$.

4) Maximum rate change of $\mathbf{f}$ : As soon as $\alpha>0$ the controller requires very high dynamics of the control output (see Fig. $5-f_{1} \ldots f_{6}$ at time $15.2 \mathrm{~s}$ ). In the simulation we imposed a realistic rate of change of the propeller speed based on experimental data. The system remains perfectly stable with the addition of this non-ideality. Although the system is stable, a smother control input directly from controller would be desirable. One option could be to limit 

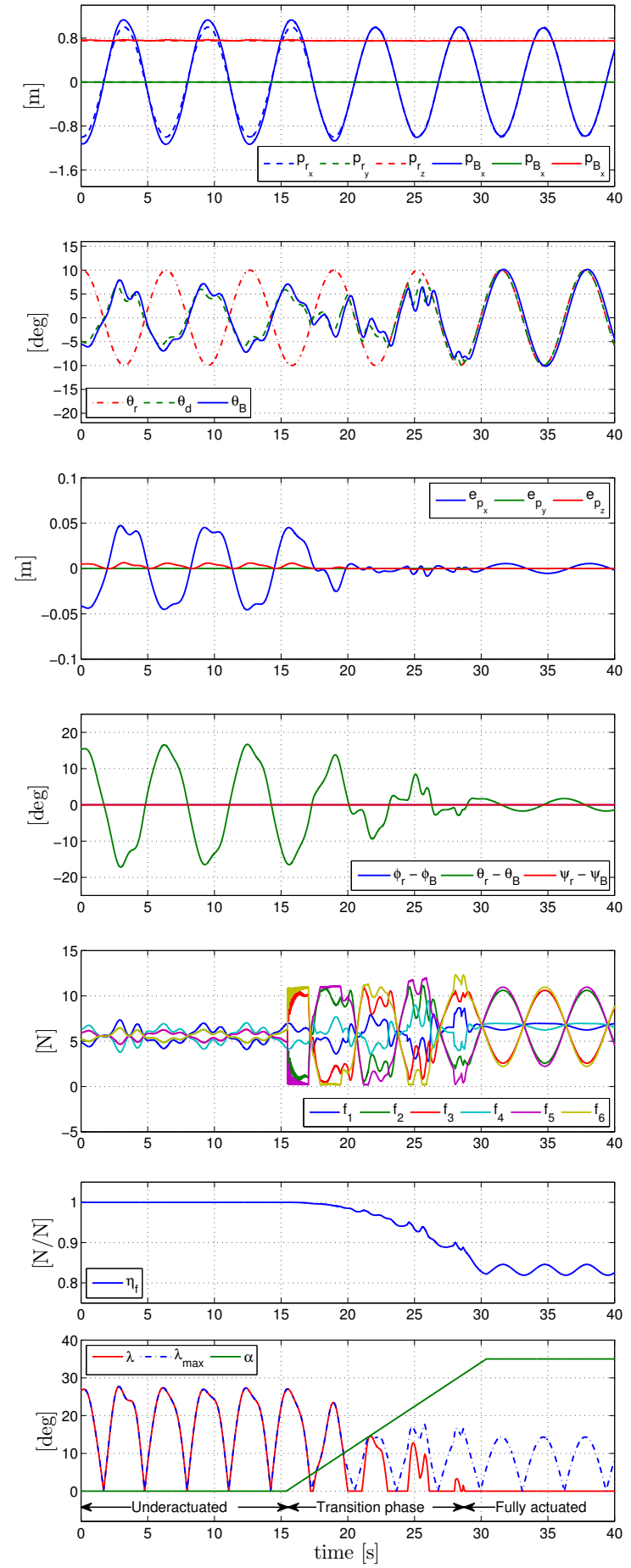

Fig. 5: Simultaneous tracking of a sinusoidal reference position trajectory and an opposing-phase reference sinusoidal attitude trajectory. What is impossible for a standard underactuated platform becomes feasible for the FAST-Hex when $\alpha$ becomes large enough. The proposed geometric controller works seamlessly in any $\alpha$ condition. All symbols are defined in the paper.

the rate of change when searching for $\lambda$ in the position controller in (21) thus achieving a continuous and smooth control output $\mathbf{u}$. This is left as future work.

\section{COnClusion And Future Work}

In this paper we presented a new multi-rotor aerial concept, named FAST-Hex, that is able to smoothly change between an efficient but underactuated aerial platform and a fully-actuated but less efficient one. Additionally, we also proposed a new full-pose geometric controller in $\mathbb{R}^{3} \times \mathrm{SO}(3)$ for generic multi-rotor platforms that is able to cope with propeller spinning rate saturation. Finally we have shown the practicability of this method for controlling the FASTHex with realistic simulations.

In the near future we will build a real prototype and test the controller with real hardware. Sensor-based automatic calibration procedures will be designed, as, e.g., in [15]. Furthermore, we will improve the controller to explicitly cope with limitation of the propeller spinning acceleration (not only the rate); we will work on the optimal and automated control of $\alpha$ and the control of the platform in the case of motor failures.

\section{REFERENCES}

[1] G. Gioioso, M. Ryll, D. Prattichizzo, H. H. Bülthoff, and A. Franchi, "Turning a near-hovering controlled quadrotor into a 3D force effector," in 2014 IEEE Int. Conf. on Robotics and Automation, Hong Kong, China, May. 2014, pp. 6278-6284.

[2] B. Yüksel, C. Secchi, H. H. Bülthoff, and A. Franchi, "A nonlinear force observer for quadrotors and application to physical interactive tasks," in 2014 IEEE/ASME Int. Conf. on Advanced Intelligent Mechatronics, Besançon, France, Jul. 2014, pp. 433-440.

[3] P. Stegagno, M. Basile, H. H. Bülthoff, and A. Franchi, "A semiautonomous UAV platform for indoor remote operation with visual and haptic feedback," in 2014 IEEE Int. Conf. on Robotics and Automation, Hong Kong, China, May. 2014, pp. 3862-3869.

[4] R. Voyles and G. Jiang, "A nonparallel hexrotor UAV with faster response to disturbances for precision position keeping," in 2014 IEEE Int. Symp. on Safety, Security and Rescue Robotics, West Lafayette, IN, Oct. 2014, pp. 1-5.

[5] P. Segui-Gasco, Y. Al-Rihani, H.-S. Shin, and A. Savvaris, "A novel actuation concept for a multi rotor UAV," in 2013 Int. Conf. on on Unmanned Aircraft Systems, Atlanta, GA, May 2013, pp. 373-382.

[6] B. Crowther, A. Lanzon, M. Maya-Gonzalez, and D. Langkamp, "Kinematic analysis and control design for a nonplanar multirotor vehicle," AIAA Journal of Guidance, Control, and Dynamics, vol. 34, no. 4, pp. 1157-1171, 2011.

[7] M.-D. Hua, T. Hamel, P. Morin, and C. Samson, "Control of VTOL vehicles with thrust-tilting augmentation," Automatica, vol. 52, pp. 17, 2015.

[8] M. Ryll, H. H. Bülthoff, and P. Robuffo Giordano, "A novel overactuated quadrotor unmanned aerial vehicle: modeling, control, and experimental validation," IEEE Trans. on Control Systems Technology, vol. 23, no. 2, pp. 540-556, 2015.

[9] S. Rajappa, M. Ryll, H. H. Bülthoff, and A. Franchi, "Modeling, control and design optimization for a fully-actuated hexarotor aerial vehicle with tilted propellers," in 2015 IEEE Int. Conf. on Robotics and Automation, Seattle, WA, May 2015, pp. 4006-4013.

[10] Y. Long, L. Wang, and D. J. Cappelleri, "Modeling and global trajectory tracking control for an over-actuated MAV," Advanced Robotics, vol. 28, no. 3, pp. 145-155, 2013.

[11] A. Oosedo, S. Abiko, S. Narasaki, A. Kuno, A. Konno, and M. Uchiyama, "Flight control systems of a quad tilt rotor unmanned aerial vehicle for a large attitude change," in 2015 IEEE Int. Conf. on Robotics and Automation, Seattle, WA, May 2015, pp. 2326-2331.

[12] D. Brescianini and R. D'Andea, "Design, modeling and control of an omni-directional aerial vehicle," in 2016 IEEE Int. Conf. on Robotics and Automation, Stockholm, Sweden, May 2015.

[13] T. Lee, M. Leoky, and N. H. McClamroch, "Geometric tracking control of a quadrotor UAV on SE(3)," in 49th IEEE Conf. on Decision and Control, Atlanta, GA, Dec. 2010, pp. 5420-5425.

[14] R. Mahony, S.-H. Cha, and T. Hamel, "A coupled estimation and control analysis for attitude stabilisation of mini aerial vehicles," in 2006 Australasian Conf. on Robotics \& Automation, Auckland, New Zealand, Nov. 2006, pp. 3003-3012.

[15] A. Censi, A. Franchi, L. Marchionni, and G. Oriolo, "Simultaneous maximum-likelihood calibration of odometry and sensor parameters," IEEE Trans. on Robotics, vol. 29, no. 2, pp. 475-492, 2013. 